\title{
Comparability of three spectrometers for monitoring urban aerosol
}

\author{
A. Khlystova, , G.P.A. Kos ${ }^{\mathrm{a}}$, H.M. ten Brink ${ }^{\mathrm{a}, *}$, A. Mirme ${ }^{\mathrm{b}}$, Th. Tuch ${ }^{\mathrm{c}, 2}$, \\ Ch. Roth ${ }^{\mathrm{c}}$, W.G. Kreyling ${ }^{\mathrm{c}}$ \\ ${ }^{a}$ Netherlands Energy Research Foundation (ECN), P.O. Box 1, Westduinweg 3, 1755 ZG Petten, The Netherlands \\ ${ }^{\mathrm{b}}$ University of Tartu, Tartu, Estonia \\ ${ }^{\mathrm{c}}$ GSF - National Research Center for Environment \& Health, Institute for Inhalation Biology, D-85758 Neuherberg/Munich, Germany
}

Received 26 May 2000; received in revised form 10 October 2000; accepted 18 October 2000

\begin{abstract}
The comparability was tested of three aerosol "spectrometers", used in a program for monitoring the spectra of fine and ultrafine particles in three European cities. Droplets of sebacate, solid ammonium sulfate and agglomerates of elemental carbon were used in the tests, representing the major chemical and structural types of particles encountered in urban aerosol. Particles in the ultrafine range $(10-100 \mathrm{~nm}$ ) are sized by electrical mobility (SMPS, DMPS and EAS) and the "spectrometers" gave very similar size distributions for these aerosols. The integrated number concentrations were on average within $20 \%$ of the directly measured total number concentrations. Particles with a size between 0.1 and $2.5 \mu \mathrm{m}$, in which most of the volume/mass is concentrated, are being differently classified in the three "spectrometers", respectively, with a low- and a high-flow LAS-X, and field charging in the EAS. The agreement between the three instruments in this size range was less good, which was partly caused by signal overload in the high-flow optical sizer, which was solved using a larger threshold. A complication occurred with the elemental carbon, which was composed of highly agglomerated entities. Particles, sized by the mobility instrumentation as being in the range of $100-400 \mathrm{~nm}$, were not detected by the optical sizers. Volume (spectra) for ammonium sulfate deduced from the number spectra were compared with the mass (spectra) obtained with cascade impactors. The comparison was good for the LAS-Xs; the EAS overestimated volume/mass. (C) 2001 Elsevier Science Ltd. All rights reserved.
\end{abstract}

Keywords: Ultrafine aerosol; Monitoring; Optical size; Mobility

\section{Introduction}

Human exposure and response to urban particles of different size is being assessed in a concerted European project, see acknowledgement. The reason behind measurement of the size of the particles is that the aerosol

\footnotetext{
${ }^{1}$ Also at: Department of Engineering and Public Policy, Carnegie Mellon University, Pittsburgh, PA 15213, USA.

* Corresponding author. Tel.: + 31-224-56-41-48; fax: + 31224-56-34-88.

${ }^{2}$ Also at: Department of Chemistry and Biochemistry, University of Maryland, College Park, MD 20740, USA.

E-mail address: tenbrink@ecn.nl (H.M. ten Brink).
}

parameter best related to the health-effect have not been identified yet. This may be mass or number of particles as was recently demonstrated by Peters et al. (1997), or surface. Health effects presumably depend on the location at which the particles are preferentially deposited in the respiratory system, which is a function of their size. Particles which dominate the number concentration are in the so-called ultrafine size range, i.e. have diameters smaller than $100 \mathrm{~nm}$. It has been shown that the human defense mechanism is less adapted in combating particles of this size (Oberdörster et al., 1995).

There are presently no commercial "spectrometers" for long-term measurement of aerosol and, therefore, homebuilt instruments or modified research instruments are used. While calibration of the size is easy using reference 
monodisperse particles, such "spectrometers" cannot be calibrated in the field with respect to the aerosol number in a size class. Without a possibility of absolute calibration the best alternative is to test the comparability of "spectrometers" side-by-side using the same aerosol, thus guaranteeing that aerosol data from different cities may indeed be compared.

Simulated urban aerosol was used in a first test of the "spectrometers", for the following reasons:

- The physical and chemical properties of the generated aerosols are well known and stable over time, so that differences in response of the instruments can only be due to aerosol composition or structure. Also extreme conditions can be chosen with respect to number/size and composition.

- Tests can be repeated later, under identical or slightly modified conditions, to systematically search for the cause of the differences.

Three kinds of aerosol material were used, representing the three major chemical components in urban aerosol. Elemental carbon represented "soot", an organic compound (bis-(2-ethylhexyl)-sebacate oil) simulated the class of organic carbonaceous compounds, while ammonium sulfate represented the dominant species of regional haze (ten Brink et al., 1997). The components also represent three different physical types of aerosol. The sebacate consists of spherical droplets, ammonium sulfate is composed of solid crystals and the elemental carbon particles are agglomerates of numerous ultrafine primary particles.

The mass distribution cannot be reliably monitored with high time resolution and, therefore, the volume as a function of size, derived from the size spectra, is used as a proxy/surrogate (Tuch et al., 1997; Eldering and Glasgow, 1998). The consistency between the volume distribution measured with the "spectrometers" and the mass distribution derived by other means was therefore also tested here.

Note: Different "spectrometers" provide aerosol number (concentrations) in different size bins. The continuous distribution of the number concentration as a function of size, constructed from the number concentration per discrete size class, can be most easily compared. This continuous function is called number "spectrum" here. The volume spectrum is defined in a similar way.

\section{Methods}

\subsection{Aerosol instrumentation}

\subsubsection{Aerosol "spectrometers"}

The three "spectrometers" tested were: the MAS (of the partners from Munich), the DAS (Dutch Aerosol "spectrometer") which is similar to MAS and the $E A S$ of the Estonian/Finnish partners, which is a completely different instrument. Performance, calibration and maintenance of MAS and EAS have been described in a previous paper (Tuch et al., 2000) and only features relevant for the present tests are given here.

The MAS consists of a differential mobility analyzer (DMA, EC-3071 TSI Inc.) - CPC (3761, TSI Inc.) combination, and an optical particle sizer (LAS-X, PMS Inc.). The LAS-X, which is a specially designed low-flow instrument, is "mobility calibrated" using aerosol which is size-classified with the DMA of the DMPS. The spectral data obtained with DMPS and the mobility-calibrated LAS-X thus, provide a single "mobility" size distribution over the diameter range of $10 \mathrm{~nm}$ to $2.5 \mu \mathrm{m}$. The time resolution of MAS (as well as the DAS, see below) is $6 \mathrm{~min}$, so that five spectra are obtained per test aerosol. The mobility calibration method failed for the EC-particles, see results.

In the Dutch "spectrometer" the scanning mode of operation of the DMA is used (SMPS 3934, TSI Inc.) with a CPC 3010 as counter. The LAS-X optical sizer is the standard clean-room high-flow type which means that it may have overload problems with high number concentrations. The aerosol is in principle classified into 16 size classes instead of into 64 channels as is done in the Munich "spectrometer". The first two channels of the LAS-X are not being used because of too low counting efficiency (Yoo et al., 1996); particles in the range $100-175 \mathrm{~nm}$ are sized with the SMPS instead.

The EAS (Kikas et al., 1996) consists of two electrical mobility analyzers, one for particles $<0.5 \mu \mathrm{m}$ and one for particles $>0.3 \mu \mathrm{m}$. The size resolution of the EAS is 4 channels per size decade. The mobility analyzer for the smaller size range uses unipolar diffusion charging (different from the bipolar charging in DAS and MAS), while the second analyzer uses electric field charging. The deflected, charged, particles are collected on the cylindrical outer walls and the current induced in discharging the walls is measured and translated to particle number. The full size spectrum is obtained by numerical inversion of the mobility distribution obtained with the two different analyzers of the EAS (Kikas et al., 1996). The EAS measures a spectrum every $10 \mathrm{~min}$, providing three spectra per test.

\subsubsection{Condensation particle counters}

Two condensation particle counters (TSI type 3022) measured total number concentrations, which were compared with the integrated number concentrations obtained from the "spectrometers". The $50 \%$ counting efficiency of the CPCs is at about $7 \mathrm{~nm}$ (Quant et al., 1992), so that they effectively measure all particles used in the present tests.

\subsubsection{Volume versus mass}

Mass spectra between 0.06 and $8 \mu \mathrm{m}$ were obtained by collecting aerosol with (two) Berner eight-stage cascade 
low-pressure impactors. The mass spectra were compared with the volume spectra derived from the "spectrometers". Ammonium sulfate aerosol was used and the amount of collected material was measured with standard analytical techniques. The mass distribution of the test aerosol was similar that in ambient air (ten Brink et al., 1997), but the associated number of particles was higher to obtain sufficient material for analysis. In ten Brink et al. (2000) details of the mass measurements are provided.

\subsection{Aerosol generation}

For each of the three species chosen two distributions were produced, one "small" and one "large". The design median diameter in number was $30-40 \mathrm{~nm}$ for the "small" particles and $70 \mathrm{~nm}$ for the "large" aerosol.

Ammonium sulfate: This aerosol was produced by nebulizing aqueous solutions with a Wiesbadener jetnebulizer, chosen for its stable output (ten Brink et al., 2000). "Small" and "large" aerosol spectra were produced using solutions with concentrations of 0.05 and $2.5 \mathrm{~g} \mathrm{l}^{-1}$, respectively. The aerosol was dried with a 10 -fold excess of dry air $(\mathrm{RH}<10 \%)$. The aerosol was then led into the test circuit described below.

Sebacate: (Bis-(2-ethylhexyl)-sebacate) was produced in a similar way as the ammonium sulfate by nebulizing ethanol solutions, with concentrations of 2 and $10 \mathrm{mll}^{-1}$ of ethanol for the "small" and "large" spectra, respectively.

Elemental carbon: It was produced with a commercial generator (GFG 1000, Palas, Karlsruhe, Germany). The generator operates by a spark-discharge between graphite rods. The structure of the aerosols is similar to that of "soot" particles in diesel exhausts.

Aerosol of ammonium sulfate and EC was stable within about $3 \%$ over a test period of 30 min with respect to total number concentration, whereas sebacate had 5\% random variations.

\subsection{Aerosol circuit}

The tests were performed in the ECN humidograph (ten Brink et al., 2000), which is especially suitable for intercomparison campaigns because of its high flow rate $\left(4001 \mathrm{~min}^{-1}\right)$. The flow in the system is well mixed, so that there is little difference in aerosol concentration across the exit plane, making the positioning of the sampling inlets less critical for the comparison. The total number concentrations were comparable with that in urban air: $3 \times 10^{4} \mathrm{~cm}^{-3}$. For each test data were collected for a period of $30 \mathrm{~min}$.

\section{Results and discussion}

An overview of the results is provided in Table 1: number geometric mean diameter, geometric standard deviation and integrated number concentrations per test, which allows a more quantitative comparison. It can be seen that the three "spectrometers" give very comparable size distributions for the ultrafine particles, i.e. for particles smaller than $100 \mathrm{~nm}$ in diameter. As an example the spectrum of the "small" elemental carbon is shown in Fig. 1.

The integrated number concentrations (Table 1b) show differences of up to $30 \%$ for an individual case, but the spectrometers agreed on average within $10 \%$. The integrated number concentration also compared well with the total number concentration directly obtained with the CPC's. Table $1 \mathrm{~b}$ shows that these are on average equal within $20 \%$ except for the large EC-particles, which will be discussed below. There is obviously no systematic difference between the aerosol spectrometers, which gives confidence in the comparability of data gained at the different ambient sites at which they have been subsequently been operated (Ruuskanen et al., 2001).

Note: Some of the differences between the monitors are indicative of their limited accuracy. For instance, the two relatively simple CPCs showed random differences of up to $30 \%$ (Table $1 \mathrm{~b}$ ), whereas the manufacturer indicates that this type of CPC has sample flow variations of up to $10 \%$.

Hereafter, we will focus on discussing measurement of particles with a diameter larger than $100 \mathrm{~nm}$, because for those particles sometimes, large discrepancies between the spectrometers were observed.

Sebacate: The "large" spectrum had a substantial number of particles larger than $100 / 175 \mathrm{~nm}$, which are sized by the LAS-X monitors in MAS and DAS, respectively. The high-flow LAS-X suffered from electronic overloading by the high particle number. It should be noted that the LAS-X does not issue a warning for this. The present finding was the reason to use a higher threshold diameter (of $275 \mathrm{~nm}$ ) in the DAS in the field monitoring program. However, measurements of the urban aerosol of Erfurt with its rather high total number concentrations show that the contribution of particles with a diameter larger than $275 \mathrm{~nm}$ is rather low (Tuch et al., 1997). Consequently, the DAS is not expected to experience serious overload. Moreover, the volume spectra deduced from the size spectra of the DAS are not affected by the overloading, see below.

The LAS-X of the MAS was not overloaded because it is one of the few instruments for measurement of high number concentrations. On the other hand, this LAS-X measured a concentration which was higher than that obtained with DMPS/SMPS in the same size range.

Ammonium sulfate: All three spectrometers (Table 1) give an almost identical shape of the spectrum.

Elemental carbon: While DAS and EAS indicate that there is a substantial number of particles in the size range of $100-300 \mathrm{~nm}$ (Fig. 2a), the MAS counts very few 
Table $1^{\text {a }}$

(a) Number geometric mean diameter $d_{\mathrm{p}}$ and geometric standard deviation $\sigma_{\mathrm{g}}$ of aerosol spectra for the different aerosol types (number geometric mean diameter is expressed in $\mathrm{nm}$ )

\begin{tabular}{|c|c|c|c|c|c|c|}
\hline & MAS & & EAS & & DAS & \\
\hline & $d_{\mathrm{p}}(\mathrm{nm})$ & $\sigma_{\mathrm{g}}$ & $d_{\mathrm{p}}(\mathrm{nm})$ & $\sigma_{\mathrm{g}}$ & $d_{\mathrm{p}}(\mathrm{nm})$ & $\sigma_{\mathrm{g}}$ \\
\hline AS-small & 25 & 1.64 & 32 & 1.86 & 27 & 1.7 \\
\hline AS-large & 55 & 2.21 & 62 & 2.38 & 54 & 2.27 \\
\hline EC-small & 35 & 1.66 & 44 & 1.86 & 38 & 1.78 \\
\hline EC-large & 76 & 1.96 & 92 & 1.64 & 80 & 1.55 \\
\hline SE-small & 76 & 1.96 & 93 & 2.09 & 70 & 1.73 \\
\hline SE-large & 213 & 2.08 & 229 & 2.08 & 131 & 2.25 \\
\hline
\end{tabular}

(b) Average total number concentrations $\left(\mathrm{cm}^{-3}\right)$ measured with the individual instruments

\begin{tabular}{|c|c|c|c|c|c|c|}
\hline & & MAS & EAS & DAS & CPC-1 & CPC-2 \\
\hline \multirow{2}{*}{ AS-small } & Aver. & 39,100 & 29,900 & 39,700 & 28,700 & 28,200 \\
\hline & Std & 1970 & 190 & 1450 & 280 & 210 \\
\hline \multirow[t]{2}{*}{ AS-large } & Aver. & 25,700 & 26,000 & 25,700 & 27,500 & 23,100 \\
\hline & Std & 970 & 660 & 370 & 300 & 760 \\
\hline \multirow[t]{2}{*}{ EC-small } & Aver. & 39,200 & 37,600 & 35,100 & 31,000 & 30,000 \\
\hline & Std & 910 & 370 & 330 & 310 & 320 \\
\hline \multirow[t]{2}{*}{ EC-large } & Aver. & $12,000^{\mathrm{b}}$ & 18,900 & 19,100 & n.a. & 13,500 \\
\hline & Std & 460 & 140 & 350 & & 390 \\
\hline \multirow[t]{2}{*}{ SE-small } & Aver. & 20,500 & 21,400 & 33,700 & 25,200 & n.a. \\
\hline & Std & 1790 & 1000 & 1020 & 870 & \\
\hline \multirow[t]{2}{*}{ SE-large } & Aver. & 19,900 & 28,300 & 21,400 & 20,500 & 16,100 \\
\hline & Std & 950 & 1020 & 1650 & 980 & 600 \\
\hline
\end{tabular}

${ }^{\mathrm{a}} \mathrm{AS}$ - ammonium sulfate, EC - elemental carbon, SE - sebacate.

${ }^{\mathrm{b}}$ When the DMPS data are used instead of the LAS-X in the range of $0.1-0.4 \mu \mathrm{m}$, see text, the integrated number concentration is $17,800 \mathrm{~cm}^{-3}$.

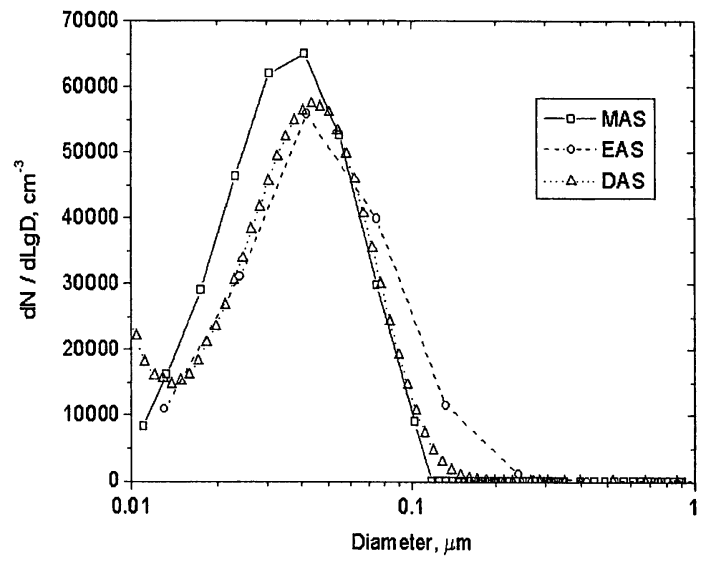

Fig. 1. Number concentration spectra for the "small" elemental carbon aerosol as measured with the three spectrometers.

particles in this region. The response of the Dutch LAS$X$, of which the data in the size range $100-275 \mathrm{~nm}$ are normally disregarded, was similarly low as that of the
LAS-X of the MAS in this region (Fig. 2b). The DMPS of the German spectrometer, which is normally not used in this size range, gave data which are in excellent agreement with that of DAS and EAS (Fig. 2b).

Schmidt-Ott and Wustenberg (1995) showed, using a high-sensitivity LAS, with a lower threshold that the optical diameter was approximately five times smaller than the mobility diameter of the same particle of agglomerates of EC generated by a spark generator of similar size. It empirically explains the present finding that the EC-particles which are seen as particles with a diameter of up to $400 \mathrm{~nm}$ by DMPS, SMPS and EAS (Fig. 2) are optically smaller than the threshold size for optical detection here of $100 \mathrm{~nm}$. The finding can be qualitatively explained using the concept of "volume equivalent" size which is the diameter size of a spherical particle with the same volume as the aggregate. Because of its highly extended geometry an aggregate has a much lower mobility than the volume equivalent particle. Hence, aggregates are classified in mobility sizing as particles which are (much) larger than the volume equivalent counterpart. 

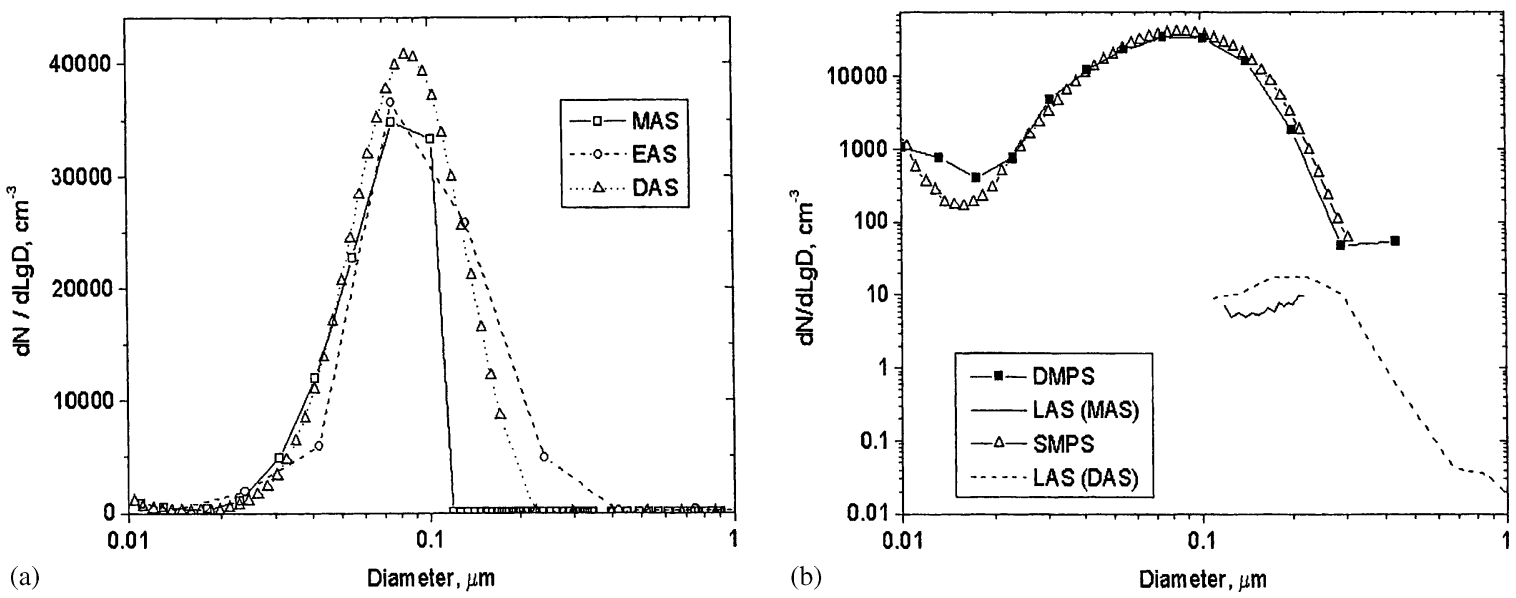

Fig. 2. Number concentration spectra for the "large" elemental carbon aerosol: (A) as measured with the standard spectrometers (B) including DMPS and high-flow LAS-X.

In optical sizing, the signal is proportional to the square of the particle volume, irrespective of the shape, as long as the geometrical extension of a particle is appreciably smaller than the wavelength of the probe light. Considering the scattered light as the sum of the scattered light of each individual primary particle within the aggregate particle, the intensity will be less than the intensity of the light scattered by a compact particle. However, for particles of the size used here, also, Mie interference from the various parts within the aggregate particle needs to be taken into account. This interference increases with the geometrical size and is, therefore, larger in the actual extended agglomerate than in the compacted volume equivalent particle. Hence, the aggregate is optically appreciated as a smaller particle.

Another complication was that the spectrometers gave integrated number concentrations which were $40 \%$ higher than those measured with the CPCs. This difference may also be caused by the large surface area of the agglomerates which can carry more electrical charge than an equivalent spherical particle. In this way, the number of particles measured in DMPS and SMPS can be overestimated. However, such extra charging was not observed for agglomerates with similar small primary nuclei (Rogak and Flagan, 1992). A more likely reason for the extra charge could be that the agglomerates are so highly charged in the generation process that they are incompletely neutralized in the spectrometers. To assess this, extra tests were made at a later time in which the EC particles were passed through an additional high-efficiency neutralizer (a large $\mathrm{Kr}-85$ neutralizer, TSI 3054) before entering spectrometer and CPC. With this extra neutralizer the spectrally integrated number concentration became, indeed, virtually equal to the total number concentration.

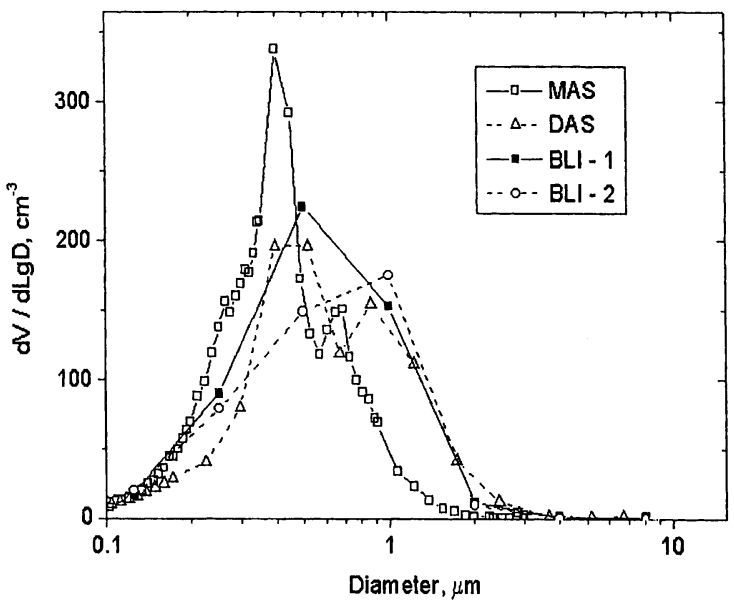

Fig. 3. The volume spectra as deduced from the number spectra for two of the three aerosol spectrometers and the volume/mass spectra obtained with two Berner low-pressure cascade impactors (B-LPI). The mass has been translated to volume with the assumption that the aerosol has the specific density of the ammonium sulfate used; the aerodynamic size of the particles has been similarly translated to volume equivalent size.

\subsection{Volume versus mass}

Volume spectra were derived from the size spectra and for some special tests, as described in the experimental section the associated mass spectra were obtained with two cascade impactors. The spectra are shown in the same graph (Fig. 3). The mass has been translated to 
volume assuming the aerosol has the specific density of the ammonium sulfate used; the aerodynamic size of the particles has been similarly translated to volume equivalent size. The volume spectra from MAS and DAS agree quite well in shape and compare well with the mass spectra. The EAS indicates quite a coarser spectrum.

A detailed analysis of the comparability of the volume and mass spectra was not made here for the following reasons. The size resolution of impactors is limited (Fig. 3) and another complication is that the impactor selects the aerosol by aerodynamic size. This is not known for ambient aerosol. Because of these problems the comparison between mass and volume was made differently, viz., by comparing the integrated mass with the integrated volume. This procedure is similar to that followed in the comparison of volume and mass of ambient aerosol (e.g. Tuch et al., 1997). The integrated mass is obtained by summing the mass collected on the various impactor stages. The integrated mass is a very precise number. Mass and volume should be related by the specific density of the aerosol; in this case $1.8 \mathrm{~g} \mathrm{~cm}^{-3}$. The experimental ratio was indeed close to 1.8 for MAS and DAS, see also ten Brink et al. (2000). The ratio deduced from the EAS was four times smaller.

\section{Concluding remarks}

Good spectral comparability in the ultrafine range was found for spectrometers used in a European exposure project, measuring aerosol of very different chemical composition and physical structure. Also the integrated number concentrations agreed well and were very comparable with the total number concentrations directly measured with condensation particle counters.

Some limitations in the instrumentation were revealed when measuring larger particles: differences in the sizing of highly agglomerated particles by the optical sizers and mobility analyzers in the spectrometers and an overload artifact in the high-flow optical sizer in case of high numbers of particles. This latter complication was solved by increasing the size threshold.

Good comparability was found between the directly measured mass (distribution) and the volume (distribution) derived from the size spectra measured with two of the three spectrometers. Consequently, the volume distribution derived from those two spectrometers appear to be a good proxy for following diurnal changes in mass distribution in urban air.

Corollary: In the field, the ratio of the number of particles measured with LAS-X and DMPS/SMPS in the overlapping size range $(0.1-0.4 \mu \mathrm{m})$ could be low, similar to the low ratio in the test here with the highly agglomerated EC-particles. Such a low ratio would then be indicative of the presence of a large number of soot particles which are highly aggregated. Analyzing the large set of observations from Tuch et al. (1997), a ratio significantly differing from one was never found.

\section{Acknowledgements}

The study was performed within the framework of the "Exposure and risk assessment for fine and ultrafine particles in ambient air" (ULTRA)-project. The project was funded by the EU ENVIRONMENT and CLIMATE Research Programme Contracts ENV4CT95-0205 and ENV4-CT97-0568. The project was coordinated by the Unit of Environmental Epidemiology, National Public Health Institute, P.O. Box 95, 70701 Kuopio, Finland.

\section{References}

Eldering, A., Glasgow, R.M., 1998. Short-term particulate matter mass and aerosol-size distribution measurements: transient pollution episodes and bimodal aerosol-mass distributions. Atmospheric Environment 32, 2017-2024.

Kikas, Ü., Mirme, A., Tamm, E., Raunemaa, T., 1996. Statistical characteristics of aerosol in Baltic Sea region. Journal of Geophysical Research 101 (D14), 19319-19327.

Oberdörster, G., Gelein, R., Ferin, J., Weiss, B., 1995. Association of particulate air pollution and acute mortality: involvement of ultrafine particles? Inhalation Toxicology 7 , $111-124$

Peters, A., Wichmann, H.E., Tuch, Th., Heinrich, J., Heyder, J., 1997. Respiratory effects are associated with the number of ultrafine particles. American Journal of Respiratory and Critical Care Medicine 155, 1376-1383.

Quant, F.R., Caldow, R., Sem, G.J., Addison, T.J., 1992. Performance of condensation particle counters with three continuous-flow designs. Journal of Aerosol Science 23, S405-S408.

Rogak, S.N., Flagan, R.C., 1992. Bipolar diffusion charging of spheres and agglomerate aerosol particles. Journal of Aerosol Science 23, 693-710.

Ruuskanen, J., Tuch, Th., ten Brink, H., Peters, A., Khlystov, A., Mirme, A., Kos, G., Brunekreef, B., Wichmann, H., Buzorius, G., Vallius, M., Kreyling, W., Pekkanen, J., 2001. Concentrations of ultrafine, fine and PM2.5 particles in three European cities. Atmospheric Environment (in press).

Schmidt-Ott, A., Wustenberg, J., 1995. Equivalent diameters of non-spherical particles. Journal of Aerosol Science 26 (Suppl. 1), S923-S924.

ten Brink, H.M., Kruisz, C., Kos, G.P.A., Berner, A., 1997. Size/composition of the light-scattering aerosol in the Netherlands. Atmospheric Environment 31, 3955-3962.

ten Brink, H.M., Khlystov, A., Kos, G.P.A., Tuch, T., Roth, C., Kreyling, W., 2000. A high-flow humidograph for testing the water uptake by ambient aerosol. Atmospheric Environment 34, 4230-4291.

Tuch, Th., Brand, P., Wichmann, H.E., Heyder, J., 1997. Variation of particle number and mass concentration in various size ranges of ambient aerosols in Eastern Germany. Atmospheric Environment 31, 4193-4197. 
Tuch, Th., Mirme, A., Tamm, E., Heinrich, J., Heyder, J., Brand, P., Wichmann, H.E., Pekkanen, J., Kreyling, W.G., 2000. Comparison of two particle size spectrometers for ambient aerosol measurements in environmental epidemiology. Atmospheric Environment 34, 146-169.
Yoo, S.-H., Chae, S.-K., Liu, B.Y.H., 1996. Influence of particle refractive index on the lower detection limit of light scattering aerosol counters. Aerosol Science and Technology 25, $1-10$. 